\title{
OEG Publication
}

Gómez-Pérez A, Ramos JA

\section{Semantic mappings: out of ontology world limits}

\author{
$1^{\text {st }}$ International Workshop on Ontology Alignment and Visualization (OnAV'08) \\ $3^{\text {rd }}$ International Conference on Availability, Reliability and Security (ARES 2008) \\ $2^{\text {nd }}$ Int. Conf. on Complex, Intelligent and Software Intensive Systems (CISIS 2008) \\ IEEE Press. \\ March 4th - 7th, 2008. \\ Barcelona, Spain. \\ Pages: 907-912 \\ ISBN: 978-0-7695-3109-0
}




\title{
Semantic mappings: out of ontology world limits
}

\author{
Gómez-Pérez A., Ramos J.A. \\ Ontology Engineering Group - Universidad Politécnica de Madrid \\ \{asun,jarg\}@fi.upm.es
}

\begin{abstract}
Mappings usually relate two similar knowledge representations. Thus, we can find many examples of mappings amid thesauri, databases, ontologies (domain ontologies, top-level and domain ontologies, PSM (Problem Solving Method) and domain ontologies, linguistic and domain ontologies); additionally, we can frequently find systems with mappings that relate two different knowledge representations, for instance, databases and ontologies. All these mappings are operationally different, and are also named differently (mappings, correspondences, semantic bridges, transformations, semantic relations, functions, conversions, domainPSM relations), but is there a single definition for these concepts? Can we find common characteristics? This paper analyzes the existing definitions and representation of the term "mapping" (and related terms) in the ontology world and its semantic neighborhood and proposes a new definition and representation of "mapping" for the Semantic Web field.
\end{abstract}

\section{Introduction}

A great variety of terms exists for naming mappings because they are widely used in many and different systems. In ontology engineering, mappings can be involved in many processes of its life-cycle. In Semantic Web applications, we have observed how the use of mapping has increased during the last years. This increase has been caused by the need to integrate resources with format, access and formalism heterogeneity. As it always occurs at the beginning of a new adventure, the different initiatives create their own particular vocabulary and definitions. In this paper we have tried to compile different approaches with the idea of defining the term "mapping" for the Semantic Web area.
The paper is divided into sections. Section 2 presents an overview of mapping definitions (general mappings, mappings in structured representations and in knowledge representations). Section 3 proposes a new definition for the term mapping as it is now used in the Semantic Web. Section 4 shows some of the existing representations of mappings. Section 5 proposes a representation for Semantic Web mappings. Section 6 shows the real use case of the proposals. Section 7 presents conclusions and future works. And finally, the last sections present the acknowledgements and references.

\section{Mappings}

\subsection{General mappings}

In this section we analyze some definitions of the term mapping found in the literature which go from the more general to the most specific definitions. Thus, we start with the definition taken from the Webster Online Dictionary [24]:

"A function such that for every element of one set there is a unique element of another set"

This definition appears also in WordNet [26], though mapping here adopts a new meaning:

"The process of locating genes on a chromosome"

To find more specific definitions of mapping in different areas (except in genetics) we have used Wikipedia [Wikipedia] and thus we have:

"In Mathematics and related technical fields, it is one kind of function.

In formal logic, it is sometimes used for a functional predicated.

In computer science, it is usually a computable function, a procedure, or a table, e.g. relating a key to its value in an associative array.

In cognitive psychology, it is the relationship between a source domain and target domain, typically reflecting a conceptual metaphor." 
From this collection of definitions, we can extract the first basic idea: mapping is a relation between knowledge units.

\subsection{Mappings in structured representations}

Within the Knowledge Representation area, the first definition found in the literature was given by Park and colleagues and published in 1998; at that time they still defined mapping abstractly (in those days, the Semantic Web did not exist):

"Whatever mechanism is used to convert between structures existing in one component and analogous structures expected by another.” [20]

As we can see, this definition is sufficiently abstract to cover almost all mappings in the Semantic Web, but the term "structure" is not specific of this field. It is important to notice that this definition identifies mapping with a mechanism determined by a purpose: to convert.

Going from structures to models, we can find mappings between modeling languages. Dominguez and colleagues, have presented a new mapping model between UML and no-UML languages since UML is the most accepted object-oriented software modeling language. And to do so, they have used the following mapping (or translation) definition:

"Given two modeling languages $L$ and $L$ ', $a$ mapping refers to a method which allows a model of $L$ ' to be determined starting from a model of L" [8]

\subsection{Mappings in knowledge representations}

When the structured representations are specialized in Knowledge Representations, new mapping definitions appear.

$\mathrm{Su}$, relating two ontologies, defined mapping as:

"Given two ontologies $A$ and $B$, mapping one ontology with another means that for each concept (node) in ontology A, we try to find a corresponding concept (node), which has the same or similar semantics, in ontology B and vice verse.” [22]

Thus, we can see that in Su's definition the structures are ontologies and the elements are ontology concepts; additionally, because only two ontologies are used, the relation is bidirectional, the semantic of relation is similarity or identity, and the conversion idea is lost.

In that same year, 2002, Maedche and colleagues published an article in which they presented ontology mapping process as follows:

"An ontology mapping process is the set of activities required to transform instances of a source ontology into instances of a target ontology." [15]

We can observed that the authors picked up the conversion idea (here, transformation) of Park et al., taking it as the objective of mappings, but specifying the transformation ontology instances.

In addition, they extended the vocabulary by introducing the term "semantic bridge" for mappings whose transformation is not equivalent:

"First, the mapping must define the two ontologies being mapped. Additionally, one may specify top-level semantic bridges which serve as entry points for the translation even if there are not mandatory. In this case the translation engine starts executing the Individual-Individual bridge.” [15]

In 2003, Crubézy and Musen presented the definition of the ontology mapping that they used in their systems:

"Our mapping ontology provides the basis for expressing the adaptation knowledge needed to configure a PSM for a certain application. In that sense, our mapping ontology extends the notion of domain-PSM bridges in the UPML framework by providing a structured and operational set of possible mapping axioms that bridge the ontologies of both components." [6]

It can be noticed that in this definition a new dimension of mapping appears: mapping between a domain ontology and a PSM (Problem Solving Method) ontology. When compared to Park's conversion and Maedche's transformation, we can observe that this definition is not classified into mappings or into semantic bridges according to the complexity of its axiom. Here mappings focus on configuring a PSM to be "executed" on concrete domain elements. Moreover, this definition does not share the object of conversion.

Euzenat and other members of the Network of Excellence Knowledge Web (FP6-507482) published a new definition of mapping between ontologies in their deliverable 2.2.1, of August of 2004:

"A formal expression that states the semantic relation between two entities belonging to different ontologies. When this relation is oriented, this corresponds to a restriction of the usual mathematical meaning of mapping: a function (whose domain is a singleton).” [2] 
Again, we can see in this definition that mapping is defined as an expression, without an explicit conversion objective. Here the definition upgrades the set of ontology components, extending Su's concept restriction, whereas the term mapping covers all complexity levels of expressions. Additionally, a new element appears in this definition: the association of a direction to mapping when the relations is a function. This direction contradicts the reciprocal definition of $\mathrm{Su}$, but this contradiction is not important since Su's only covers similarity and identity relations.

Under the term "ontology mapping” we can find other definitions of the same concept, for instance:

Kalfoglou and Schorlemmer, in their 2005 version of the state of the art of mappings, defined mapping between ontologies as:

"A morphism, which usually will consist of a collection of functions assigning the symbols used in one vocabulary to the symbols of the other." [13]

In the same article, they distinguished two types of mappings: one oriented to correspondence between representation languages and another oriented to correspondence between vocabularies. Therefore, these mappings thus defined have functions that assign terms of an ontology to terms of another ontology. This definition covers the mappings between PSM ontologies and domain ontologies. Here, mappings only relate two ontologies.

Although all definitions presented here define mappings within the Semantic Web area, none of them covers all desirable aspects (some related elements, some different representations, any relation, etc.) for being used as a general definition.

\section{Mappings in Semantic Web}

So far, we have revised almost all definitions of mappings between ontologies that have appeared in the literature and have done so in chronological order. But in the frame of the Semantic Web we need a new definition that covers the Semantic Web extension of ontologies with the properties of mappings between ontologies (these mappings are more precisely defined because of their extended use).

Ontologies are the main knowledge representation of the Semantic Web, but they are not the only ones. Integrated in the Semantic Web, we can find systems and applications whose treat with databases, natural language documents, annotated documents, web pages, semantic networks, graphs, navigation models, etc.
Therefore, these knowledge representations are susceptible of being mapped with ontologies or between them.

In addition, in the Semantic Web we can find systems that execute PSM to obtain different results with some domain ontologies. So, Semantic Web mappings need to cover directional and not-predefined functions.

The Ontology Engineering Group of the UPM (Univ. Politécnica de Madrid) have provided a new definition for mapping focused on the Semantic Web:

"A mapping is a formal explicitation of a relation between elements, or set of elements, of different conceptualizations and/or instantiations.”

This definition does not limit the relation to a reciprocal function or to declarative transformations. Thus, mappings can be established between the elements of all knowledge representations of Semantic Web and are no restricted to a number of elements or to a number of representations.

As it can be observed, this definition encompasses all the mappings that take part on different Semantic Web processes, such as ontology alignment, heterogeneous resources integration, annotation, etc.

\section{Representing mappings}

Several representations of mappings can be found in the literature:

CWM (Common Warehouse Model) [19] includes a conceptual model for generic and very expressive mappings. However, these features convert the model into a complex one. The model is composed of classes Transformation, TransformationMap, ClassifierMap, FeatureMap, ClassifierFeatureMap, TypeMapping and their properties and characteristics.

This expressively restricted model was used to develop the metaontology RDFT (RDF Transfromation) [18], which specifies a little language for DTD mappings of XML to RDF-Scheme and vice versa. In this metaontology the main class is Bridge (similar to ClassifierMap of CWM). This metaontology contains the following classes: Map, EventMap, Interface, Roles, Event2Event, DocumentMap, Bridge, XMLBridge, VocabularyMap, and RDFBirdge (for more details, see [18]).

MAFRA Semantic bridge ontology (SBO) [15] presents a mapping language described in UML, but this language only targets data transformation. 
OWL defines equivalentClass and equivalentProperty as primitives, both of which can be considered explicitations of mappings [23].

C-OWL [4] is a mapping language proposal that can express relatively simple alignments between ontologies. The constructs in C-OWL are called bridge rules, and they allow expressing a family of semantic relations between concepts/roles and individuals. COWL permits representing mappings with 8 semantic relations (equivalence, containments, overlap and their negations).

SEKT Mapping Language (OML) [7] provides a set of constructs to express mappings between classes, attributes, relations and instances of an ontology.

In addition, there are some languages, such as (SWRL [12], FLogic [14], ODL ${ }_{13}$ [5], OntomatDBImport [21], D2R [3], SKOS [16, 17], $\mathrm{R}_{2} \mathrm{O}$ [1]) permit expressing mappings. Here we have focused on the language that is more similar to our mapping concept, Alignment Format by INRIA.

The last work representing mappings and providing a mapping language is the deliverable 2.2.10 of Network of Excellence Knowledge Web ${ }^{1}$ [9]. They present a language, the Mapping Language, designed by the Ontology Matching Working Group, and an implementation that supports this language. The language is based on the Alignment Format [10].

In NeOn Project ${ }^{2}$ (FP6-027595), a Mapping Metamodel for OWL [11] has been agreed. This representation covers only mappings for expressing the context of an OWL ontology.

\section{Mapping model proposal}

Starting from common elements of the abovementioned representations and following the generalization idea of our definitions for mappings in Semantic Web, we have designed a simple representation for covering mappings and their uses in the Semantic Web. Figure 1 shows this representation.

We have chosen this simple and usable representation because a harder model, such as an ontology, needs a manager to handle instances and models.

Its main contribution is independency of the knowledge representation. Thus, we can use this design to represent mappings between ontologies, between relational databases and ontologies, between thesauri, etc.

\footnotetext{
${ }^{1}$ http://www.knowledgeweb.net

${ }^{2}$ http://www.neon-project.org
}

Besides, we can notice that a mapping relation is not limited. The mapping managers can define the relations needed.

In this representation we have included component metadata (as LastModificationDate and Reference), mainly for tracing information flow.

For making this representation usable, we have presented it as an $\mathrm{XSD}^{3}$ (XML Schema Definition) so as to make explicit the mappings in XML files following this schema.

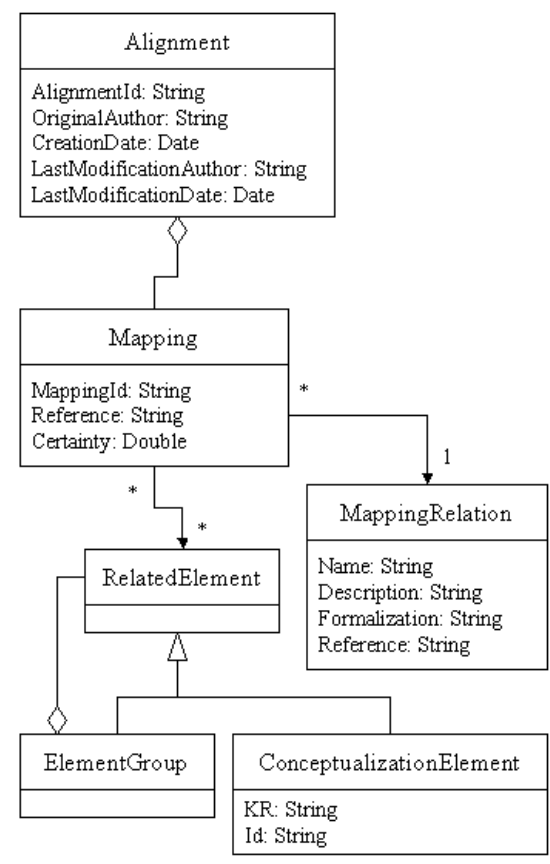

Figure 1. Mapping model proposal

\section{From proposal to reality}

Definition and representation proposals presented in this paper have been used in a bilateral agreement between the Spanish National Geographic Institute (IGN) and the UPM for integrating the current heterogeneous databases. IGN has four databases with geographic information in different scales. This information is classified into phenomena and the list of these phenomena has tremendously different granularity (for example, a catalogue has 22 phenomena and another has 560 phenomena).

UPM and IGN have jointly developed an ontology of phenomena, called PhenomenOntology, and we have planned to develop later an automatic mapping discoverer between the ontology and the relational databases. On the other hand, for managing the mappings discovered by the new tool, a Java library

\footnotetext{
${ }^{3}$ http://webode.dia.fi.upm.es/AlignmentSchema.xsd
} 
following the designed general mapping model has been developed and these mappings are serialized using the AlignmentSchema presented.

Additionally, UPM is working on the extraction of mappings of concept classification from text semantic annotations. These mappings can be used in ontology learning and in ontology aligning.

These mappings have been also represented following the mapping model proposed in this paper.

Annex I contains an example of a mapping file following AlingmentSchema XSD.

\section{Conclusions and future trends}

We have presented some proposals for defining and representing mappings in an abstract level upper than the levels they are usually dealt with, as the proposals cover all mappings in the Semantic Web.

The proposals are based on a deep analysis of the state of the art on mapping conceptualizations and representations in many systems, tools and initiatives.

Finally, we have presented the real use cases in which UPM is developing software following this model and schema.

In a near future UPM will integrate external mapping tools under this common definition and representation.

\section{Acknowledgements}

This work has been partially supported by the National Project “GeoBuddies” (TSI2007-65677C02) and the bilateral collaboration UPM-IGN 2007-2008. We are also grateful to Rosario Plaza for her help in checking and reviewing the grammar and spelling of the paper and improving clarity and style.

\section{References}

[1] Barrasa J, Corcho O, Gómez-Pérez A (2004) $\mathrm{R}_{2} \mathrm{O}$, an Extensible and Semantically Based Database-to-Ontology Mapping Language. Second Workshop on Semantic Web and Databases (SWDB2004). Toronto, Canada. August 2004. [2] Bouquet P (coordinator), Euzenat J, Franconi E, Serafini L, Stamou G, Tessaris S (2004) D2.2.1 Specification of a common framework for characterizing alignment Knowledge Web (FP6-507482). http://www.knowledgeweb.net/

[3] Bizer C (2003). D2r map - a database to rdf mapping language. In 12th Intl World Wide Web Conference Budapest May 2003.

[4] Bouquet P, Giunchiglia F, van Harmelen F, Serafini L, Stuckenschmidt H (2003) C-OWL: Contextualizing ontologies. In D. Fensel, K. Sycara, and J. Mylopoulos, editors, Proceedings of the Second International Semantic Web Conference, number 2870 in Lecture Notes in Computer Science, pages 164-179. Springer Verlag, October 2003.

[5] Buneman P, Raschid L, Ullman JD (1997). Mediator languages - a proposal for a standard. SIGMOD Record, 26(1):39-44, 1997.

[6] Crubézy M, Musen M.A. (2003) Ontologies in Support of Problem Solving. Handbook on Ontologies. S. Staab and R. Studer, Springer: 321-341.

[7] de Bruijn J, Foxvog D, Zimmerman K (2004) Ontology mediation patterns library. Deliverable D4.3.1, SEKT, 2004.

[8] Domínguez E, Rubio AL, Zapata MA (2002) Mapping Models Between Different Modeling Languages. Proceedings of the ECOOP'2002 Workshop on Integration and Transformation of UML models. J. Araujo, J. Whittle, A. Toval and R. France, Eds. Málaga, Spain. pp.18-22. June 2002.

[9] Euzenat J, Scharffe F, and Zimmermann A (2007) Expressive Alignment langaugae and implementation. Knowledge Web Deliverable 2.2.10. August 2007. http://www.knowledgeweb.net

[10] Euzenat J, Shvaiko P (2007) Ontology Matching. Springer, 2007

[11] Haase P, Brockmans S, Palma R, Euzenat J, d'Aquin M (2007) Updated Version of the Networked Ontology Model. NeOn Deliverable 1.1.2. August 2007. http://www.neonproject.org

[12] Horrocks I, Patel-Schneider P, Boley H, Tabet S, Grosof B, Dean M (2004) SWRL: a semantic web rule language combining OWL and RuleML, 2004. http://www.w3.org/Submission/SWRL/.

[13] Kalfoglou Y, Schorlemmer M. (2005) Ontology Mapping: The State of the Art, Dagstuhl Seminar on Semantic Interoperability and Integration 2005, Germany.

[14] Kifer M, Lausen G, Wu J (1990). Logical foundations of object-oriented and frame-based languages. Technical Report TR-90-003, 1, 1990.

[15] Maedche A, Motik B, Silva N, Volz R (2002). MAFRA - A MApping FRAmework for Distributed Ontologies. In Proc. of the 13th Intl. Conf. on Knowledge Engineering and Knowledge Management (EKAW'2002), volume 2473 of Lecture Notes in Computer Science, pages 235--250. Springer-Verlag.

[16] Miles A, Brickley B (2005a) Skos core guide. Technical report, World Wide Web Consortium (W3C), http://www.w3.org/TR/2005/swbp-skos-coreguide, 2005.

[17] Miles A, Brickley B (2005b) Skos core vocabulary. Technical report, World Wide Web Consortium (W3C), http://www.w3.org/TR/2005/swbp-skos-core-spec, 2005.

[18] Omelayenko B (2002) RDFT: A Mapping MetaOntology for Business Integration. In: Proceedings of the Workshop on Knowledge Transformation for the Semantic for the Semantic Web at the 15th European Conference on Artificial Intelligence (KTSW2002). Lyon, France. 2002. pp. 77-84.

[19] OMG (2001) CWM: Common Warehouse Model Specification. Technical Report, Obejct Management Group. 2001. 
[20] Park J.Y. Gennari J.H. Musen M.A. (1998) Mappings for Reuse in Knowledge-based Systems. 11 Workshop on Knowledge Acquisition, Modelling and Management KAW 98. Banff, Canada, 1998.

[21] Stojanovic L, Stojanovic S, Volz R (2002). A reverse engineering approach for migrating data-intensive web sites to the semantic web. In Proceedings of the IIP-2002 (Part of the IFIP World Computer Congress WCC2002), Montreal, Canada, 2002.

[22] Su X (2002) A Text Categorization Perspective for Ontology Mapping. http://www.idi.ntnu.no/ xiaomeng/ paper/Position.pdf

[23] Uschold M (2005) Achieving semantic interoperability using RDF and OWL - v4, 2005.

[24] Webster Online Dictionary. http://www.webstersonline-dictionary.org/

[25] Wikipedia The Free Encyclopedia. http://en.wikipedia. org/wiki/Main_Page

[26] WordNet online. http://wordnet.princeton.edu/online/

\section{Annex I}

Here we present an example of an alignment file following the proposed representation. Notice that the representation permits mappings with arity over 2 .

$<$ ?xml version="1.0" encoding="iso-8859-1"?>

<CV xmlns:xsi='http://www.w3.org/2001/XMLSchemainstance'

xsi:noNamespaceSchemaLocation='file://AlignmentSchema. xsd'>

$<$ Alignment $>$

$<$ AlignmentId $>$ AlignId11</AlignmentId $>$

$<$ OriginalAuthor $>$ Alignment Test 1.0</OriginalAuthor $>$

$<$ CreationDate>Sun Nov 11 19:05:31 CEST

$2007</$ CreationDate $>$

$<$ LastModificationAuthor $>$ Mapping discoverer

$1.0</$ LastModificationAuthor $>$

$<$ LastModificationDate>Sun Nov 11 19:15:21 CEST

$2007</$ LastModificationDate $>$

$<$ Mappings $>$

$<$ Mapping $>$

$<$ MappingId>BCN1_Fenomenos.Phenomena.030701-

PhenomenOntology.Castillo $<$ /MappingId $>$

$<$ Certainty $>1.0</$ Certainty $>$

$<$ Reference $>$ Mapping by OEG $<$ /Reference $>$

$<$ MappingRelation $>$

$<$ Name $>$ Equality $<$ /Name $>$

$<$ Description $>$ All elements are semantically

equivalents. $</$ Description $>$

$<$ Formalization $>\mathrm{A}=\mathrm{B}=\mathrm{C}=\ldots</$ Formalization $>$

$<$ Reference $>$ OEG Mapping Relations $<$ /Reference $>$

$<$ MappingRelation $>$

$<$ ConceptualizationElement $>$

$<\mathrm{KR}>\mathrm{DB}</ \mathrm{KR}>$

$<$ Id $>$ BCN1_Fenomenos.Phenomena.030701</Id $>$
$<$ ConceptualizationElement $>$

$<$ ConceptualizationElement $>$

$<\mathrm{KR}>$ Ontology $</ \mathrm{KR}>$

$<$ Id $>$ PhenomenOntology.Castillo $</$ Id $>$

$<$ ConceptualizationElement $>$

$</$ Mapping $>$

$<$ Mapping $>$

$<$ MappingId $>$ PhenomenOntology.Abadía-

BCN1_Fenomenos.Phenomena.030602</MappingId $>$

$<$ Certainty $>0.8</$ Certainty $>$

$<$ Reference $>$ Mapping by OEG $<$ /Reference $>$

$<$ MappingRelation $>$

$<$ Name $>$ Subsumption $<$ /Name $>$

$<$ Description $>$ Arity: 2 . The first element is subclass of

the second element. $</$ Description $>$

$<$ Formalization $>$ A sc $\mathrm{B}</$ Formalization $>$

$<$ Reference $>$ OEG Mapping Relations $</$ Reference $>$

$<$ /MappingRelation $>$

$<$ ConceptualizationElement $>$

$<$ KR $>$ Ontology $</$ KR $>$

$<$ Id $>$ PhenomenOntology.Abadía $</$ Id $>$

$<$ ConceptualizationElement $>$

$<$ ConceptualizationElement $>$

$<\mathrm{KR}>\mathrm{DB}</ \mathrm{KR}>$

$<$ Id $>$ BCN1_Fenomenos.Phenomena.030602</Id $>$

$</$ ConceptualizationElement $>$

$</$ Mapping $>$

$<$ Mapping $>$

$<$ MappingId $>$ BCN1_Fenomenos.Phenomena.030702-

InternalDB.Fenomenos.Fortificación-

PhenomenOntology.Fortaleza $<$ /MappingId $>$

$<$ Certainty $>0.9</$ Certainty $>$

$<$ Reference $>$ Mapping by OEG $</$ Reference $>$

$<$ MappingRelation $>$

$<$ Name $>$ Equality $<$ /Name $>$

$<$ Description $>$ All elements are semantically

equivalents. $</$ Description $>$

$<$ Formalization $>\mathrm{A}=\mathrm{B}=\mathrm{C}=\ldots</$ Formalization $>$

$<$ Reference $>$ OEG Mapping Relations $<$ /Reference $>$

$<$ /MappingRelation $>$

$<$ ConceptualizationElement $>$

$<\mathrm{KR}>\mathrm{DB}</ \mathrm{KR}>$

$<$ Id $>$ BCN1_Fenomenos.Phenomena.030702</Id $>$

$</$ ConceptualizationElement $>$

$<$ ConceptualizationElement $>$

$<$ KR $>$ Ontology $</$ KR $>$

$<$ Id $>$ PhenomenOntology.Fortaleza $</$ Id $>$

$<$ /ConceptualizationElement $>$

$<$ ConceptualizationElement $>$

$<\mathrm{KR}>\mathrm{DB}</ \mathrm{KR}>$

$<$ Id $>$ InternalDB.Fenomenos.Fortificación $</$ Id $>$

$</$ ConceptualizationElement $>$

$<$ Mapping $>$

$<$ /Mappings $>$

$</$ Alignment $>$

$</$ CV $>$ 